\title{
Stress-induced anhedonia is associated with hypertrophy of medium spiny neurons of the nucleus accumbens
}

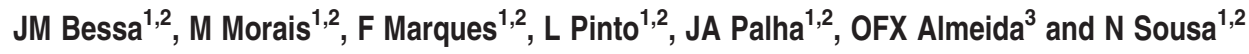

There is accumulating evidence that the nucleus accumbens (NAc) has an important role in the pathophysiology of depression. As the NAC is a key component in the neural circuitry of reward, it has been hypothesized that anhedonia, a core symptom of depression, might be related to dysfunction of this brain region. Neuronal morphology and expression of plasticity-related molecules were examined in the NAC of rats displaying anhedonic behavior (measured in the sucrose-consumption test) in response to chronic mild stress. To demonstrate the relevance of our measurements to depression, we tested whether the observed changes were sensitive to reversal with antidepressants (imipramine and fluoxetine). Data show that animals displaying anhedonic behavior display an hypertrophy of medium spiny neurons in the NAc and, in parallel, have increased expression of the genes encoding for brain-derived neurotrophic factor, neural cell adhesion molecule and synaptic protein synapsin 1. Importantly, the reversal of stress-induced anhedonia by antidepressants is linked to a restoration of geneexpression patterns and dendritic morphology in the NAc. Using an animal model of depression, we show that stress induces anhedonic behavior that is associated with specific changes in the neuronal morphology and in the gene-expression profile of the NAc that are effectively reversed after treatment with antidepressants.

Translational Psychiatry (2013) 3, e266; doi:10.1038/tp.2013.39; published online 4 June 2013

\section{Introduction}

One of the core symptoms of major depressive disorder is anhedonia (diminished interest or pleasure). ${ }^{1}$ This suggests that dysfunction of brain reward pathways contributes to the pathophysiology of the disease. Dysfunction of the nucleus accumbens (NAc), an important relay station in the brain's response to rewarding stimuli, has been consistently implicated in depression. ${ }^{2-5}$ Chronic stress is known to induce anhedonia in animal models of depression, ${ }^{6}$ and its impact on the fine structure of the NAc has been described at the synaptic and molecular levels. ${ }^{7}$

The NAc, a part of the ventral striatum, is involved in motivation, reward, learning, problem-solving and motor function, all of which are affected in clinical depression. ${ }^{8}$ The NAc, a part of the ventral striatum, consists of two functionally distinct divisions: the core and the shell. Whereas the core is associated with goal-directed behavior, instrumental learning and motivation, ${ }^{9,10}$ the shell is thought to integrate motivational valence and novelty. ${ }^{11}$ The majority of the NAc is composed of medium spiny $\gamma$-aminobutyric acid (GABA)ergic neurons, but the structure also contains smaller populations of interneurons that express GABA or acetylcholine.$^{12}$ Importantly, the NAc is part of a complex network receiving glutamatergic, dopaminergic, serotonergic, noradrenergic and cholinergic afferents that originate in brain regions sensitive to stress-induced depression. ${ }^{13-15}$ Previous studies have reported that stress-induced dendritic and synaptic plasticity in the NAc are modulated by brain-derived neurotrophic factor (BDNF) ${ }^{16}$ and the neuropeptide melanocortin. ${ }^{17}$

In the present study, we searched for the structural and molecular correlates of anhedonic behavior in the NAc of rats that had been subjected to a chronic mild stress (CMS) protocol. In addition, we examined whether the stress-induced changes in plasticity within the NAc are influenced by two commonly used antidepressants, fluoxetine and imipramine. Our results show that stress-induced anhedonic behavior is associated with changes in the morphology of dendrites of medium spiny neurons of the NAc with concomitant changes in the levels of expression of various neurotrophins, cell adhesion molecules and synaptic proteins in the NAc. Antidepressant treatment with either fluoxetine or imipramine reversed these stress-induced morphological and neurochemical alterations while restoring hedonic behavior.

\section{Materials and methods}

Animals. Male Wistar rats (Charles-River Laboratories, Barcelona, Spain), weighing 300-400 g and aged 3 months, were used in this study. Animals were housed (three per cage) under standard laboratory conditions (12 h light: $12 \mathrm{~h}$ dark cycle, at $22{ }^{\circ} \mathrm{C}$, relative humidity of $55 \%$; free access to food and water). Animals were assigned to one of the two main treatment groups (control, $n=14$ and CMS, $n=42$ ). All procedures were carried out in accordance with the

\footnotetext{
${ }^{1}$ Life and Health Science Research Institute (ICVS), School of Health Sciences, University of Minho, Braga, Portugal; ${ }^{2}$ ICVS/3B'sPT Government Associate Laboratory, Braga/Guimarães, Portugal and ${ }^{3}$ Max Planck Institute of Psychiatry, Munich, Germany

Correspondence: Dr N Sousa, Neuroscience Research Domain, Life and Health Science Research Institute (ICVS), University of Minho, Campus de Gualtar, Braga 4710-057, Portugal.

E-mail: njcsousa@ecsaude.uminho.pt

Keywords: anhedonia; antidepressants; depression; neuroplasticity; remodeling; stress

Received 18 December 2012; revised 11 April 2013; accepted 21 April 2013
} 
Portuguese national authority for animal experimentation, Direção Geral de Veterinária (ID: DGV9457) and in accordance with the guidelines for the care and handling of laboratory animals in the Directive 2010/63/EU of the European Parliament and of the Council.

Drugs. The drugs used were fluoxetine $\left(10 \mathrm{mg} \mathrm{kg}^{-1}\right.$, Kemprotec, Middlesbrough, UK) and imipramine (10 $\mathrm{mg} \mathrm{kg}^{-1}$, Sigma-Aldrich, St Louis, MO, USA). Compounds were dissolved in $5 \%$ DMSO in $0.9 \%$ saline and administered intraperitoneally $\left(1 \mathrm{ml} \mathrm{kg}^{-1}\right)$ to animals, daily at 20:00 hours, during the last 2 weeks of the CMS protocol. The doses of antidepressant drugs administered daily were chosen based on their therapeutic effects as described in the literature. ${ }^{18}$

CMS. A slightly modified version of an unpredictable CMS protocol was used. ${ }^{6}$ It consisted of chronic exposure to unpredictable mild stressors (confinement to a restricted space for $1 \mathrm{~h}$, placement in a tilted cage $\left(30^{\circ}\right)$ for $3 \mathrm{~h}$, housing on damp bedding for $8 \mathrm{~h}$, overnight illumination, food deprivation for $18 \mathrm{~h}$ followed by exposure to inaccessible food for $1 \mathrm{~h}$, water deprivation for $18 \mathrm{~h}$ followed by exposure to an empty bottle for $1 \mathrm{~h}$ and reversed light/dark cycle for $48 \mathrm{~h}$ every 7 days) over 6 weeks. During the last 2 weeks of CMS, animals were given daily injections with saline $(n=14)$, fluoxetine $(n=14)$ or imipramine $(n=14)$.

Open-field test. Locomotor activity was investigated using the open-field test in a room brightly illuminated by white light. Briefly, rats were placed in the center of an arena $\left(43.2 \times 43.2 \mathrm{~cm}^{2}\right.$, transparent acrylic walls and white floor, MedAssociates, St Albans, VT, USA) and instant position was monitored online over a period of $5 \mathrm{~min}$ with the aid of two 16beam infrared arrays. Total distances travelled and average speeds were used as measures of locomotor activity.

Sucrose-preference test. Anhedonia was assessed weekly during exposure to CMS using the sucrose preference test. Briefly, animals were allowed to habituate to the sucrose solution 1 week before the CMS protocol to establish baseline preference levels. To test sucrose preference, animals that were food- and water-deprived for $18 \mathrm{~h}$ were presented with two pre-weighed bottles containing $1 \%$ sucrose solution or tap water for a period of $1 \mathrm{~h}$. Sucrose preference was calculated according to the formula: sucrose preference $=$ (sucrose intake/(sucrose intake + water intake) $) \times 100$, as previously described. ${ }^{19}$ Anhedonia was defined as a reduction in sucrose preference relative to baseline levels.

Forced swimming test (FST). Learned helplessness was evaluated in the FST on the last day of exposure to CMS. Twenty four hours after a pre-test session (10 min), rats were placed in cylinders filled with water $\left(25^{\circ} \mathrm{C}\right.$; depth $\left.30 \mathrm{~cm}\right)$ for a period of $5 \mathrm{~min}$. Test sessions were assessed using a camera connected to a video tracking system (Viewpoint, Lyon, France); the system automatically calculated immobility time and latency to immobility. Learned helplessness behavior was defined as an increase in time of immobility and a decrease in latency to immobility.
Structural analysis. At the end of the experimental procedures, animals were killed under anesthesia. The total number of neurons in the core and shell divisions of the $\mathrm{NAc}$ was estimated using the optical fractionator ${ }^{20}$ in the right hemisphere of four animals from each experimental group. Brains were postfixed in $4 \%$ paraformaldehyde for $48 \mathrm{~h}$ and embedded in glycolmethacrylate. Volumes were determined by Cavalieri's principle using the Stereolnvestigator software (Microbrightfield, Willinston, ND, USA). For the tri-dimensional morphometric analysis, six animals from each treatment group were transcardially perfused with $0.9 \%$ saline and processed. ${ }^{21}$ Briefly, brains were immersed in Golgi-Cox solution $^{22}$ for 21 days, transferred to a $30 \%$ sucrose solution and cut on a vibratome. Coronal sections ( $200 \mu \mathrm{m}$ thick) were collected in $6 \%$ sucrose and blotted dry onto gelatin-coated microscope slides. They were subsequently alkalinized in $18.7 \%$ ammonia, developed in Dektol (Kodak, Rochester, NY, USA), fixed in Kodak Rapid Fix, dehydrated and xylenecleared before coverslipping. Dendritic arborization and spine numbers and shape were analyzed in the core and shell divisions of the NAc. For each selected neuron, all branches of the dendritic tree were reconstructed at $\times 600$ (oil) magnification using a motorized microscope (Axioplan 2, Carl Zeiss, Thornwood, NY, USA) and Neurolucida software (Microbrightfield). A 3D analysis of the reconstructed neurons was performed using the NeuroExplorer software (Microbrightfield). Forty neurons were studied for each animal and measurements from individual neurons from each animal were averaged. Several aspects of the dendritic morphology were examined: (i) total dendritic length was compared across the experimental groups; (ii) dendritic spine density (number of spines/dendritic length) was determined in branches that were either parallel or at acute angles to the coronal surface of the section; and (iii) spine morphology, specifically by classifying spines in the selected segments as mushroomshaped, thin, wide and ramified spines, with the proportion of each type of spine being calculated for each neuron. ${ }^{23}$

Gene-expression measurements by quantitative real time PCR (qRT-PCR). Levels of Bdnf, Ncam1 (neural cell adhesion molecule) and Syn1 (synapsin 1) mRNA were determined by qRT-PCR in NAc derived from four animals of each treatment group. Tissue from the NAc was dissected under the microscope. Briefly, $2 \mu \mathrm{g}$ of total RNA were reverse transcribed using oligo-dt primers of the Superscript First-strand Synthesis system for RT-PCR (Invitrogen, Carlsbad, CA, USA). Hypoxanthine guanine phosphoribosyl transferase (Hprt) was used as internal standard for normalization. Oligonucleotide primers for Bdnf (sense $5^{\prime}$-GCGG CAGATAAAAAGACTGC-3', antisense 5'-GCAGCCTTCCTT CGTGTAAC-3'), Ncam1 (sense 5'-AAAGGATGGGGAACC CATAG-3', antisense 5'-TAGGTGATTTTGGGCTTTGC-3'), Syn1 (sense 5'-CACCGACTGGGCAAAATACT-3', antisense 5'-TCCGAACTTCCATGTCC-3') and Hprt (sense 5'-GCAG ACTTTGCTTTCCTTGG-3', anti-sense 5'-TCCACTTTCGC TGATGACAC-3') were designed using the Primer3 software (Simgene, San Francisco, CA, USA) on the basis of the GenBank sequences NM_031521, NM_019133 and NM_012583, respectively. qRT-PCR was performed on a CFX 96TM real-time system instrument (Bio-Rad 
Laboratories, Hercules, CA, USA), with the QuantiTect SYBR Green RT-PCR reagent kit (Qiagen, Hamburg, Germany) according to the manufacturer's instructions, using equal amounts of RNA from each of the samples. Product fluorescence was detected at the end of the elongation cycle. All melting curves exhibited a single sharp peak at the expected temperature.

Statistical analysis. After confirmation of homogeneity, appropriate statistical tests were applied to the data. Repeated-measures analysis of variance were used to analyze the results of the sucrose consumption test (SCT). Two-factor analysis of variance was used to evaluate further behavioral, structural and gene-expression data. Differences between groups were then determined by Tukey's honestly significant difference test post-hoc analysis. Statistical significance was accepted for $P<0.05$. Results are expressed as mean \pm s.e.m.

\section{Results}

Behavioral results. Anhedonia was assessed weekly in the SCT, performed during the 6 weeks of the experimental protocol. Animals exposed to CMS revealed a significant decrease in sucrose preference when compared with control animals $\left(F_{1,26}=89.53, P<0.001\right)$ (Figure $\left.1 \mathrm{a}\right)$, indicating an anhedonic phenotype. The administration of antidepressants in the last 2 weeks of the CMS protocol significantly reversed this behavioral phenotype $\left(\mathrm{F}_{2,52}=4.875, P=0.011\right)$. As previously shown, ${ }^{24}$ the tricyclic antidepressant imipramine elicited an earlier onset of antidepressant action, reversing anhedonic behavior within the first week of treatment $(P<0.001)$, whereas the response to the selective serotonin reuptake inhibitor fluoxetine was only evident after 2 weeks $(P<0.001)$ (Figure 1a). Consistent with the SCT data, learned helplessness in the FST revealed that animals exposed to CMS showed increased immobility time $\left(F_{1,26}=37.568\right.$, $P<0.001$ ) (Figure 1b) and decreased latency to immobility $\left(F_{1,26}=32.894, P<0.001\right)$ (Figure $\left.1 c\right)$. Antidepressant treatment proved to be a significant factor in recovery from learned helplessness $\left(F_{2,52}=27.962, P<0.001\right)$; when compared with stressed rats that did not receive drug treatments, immobility time was significantly reduced by fluoxetine $(P<0.001)$ and imipramine $(P<0.001)$ and the latency time to immobility was significantly increased by both drugs $(P<0.001)$. As previously described, the concordance between the anhedonic behavior in the SCT and the learned helplessness in the FST further strengthens the consistency of the CMS protocol as an animal model of depression. ${ }^{24}$ No significant effects of CMS or treatment with fluoxetine and imipramine were observed in locomotor activity evaluated in the open-field test (Supplementary Figure S1).

Structural analysis. Volumetric measurements of the core and shell divisions of the NAc revealed a tendency for increased volume in both the subregions in animals exposed to CMS without antidepressant treatment that, however, failed to reach statistical significance (shell: $P=0.612$; core: $P=0.339$ ) (Figures $2 c$ and d). Stereological estimation of the total number of cells in the core and shell divisions of the NAc
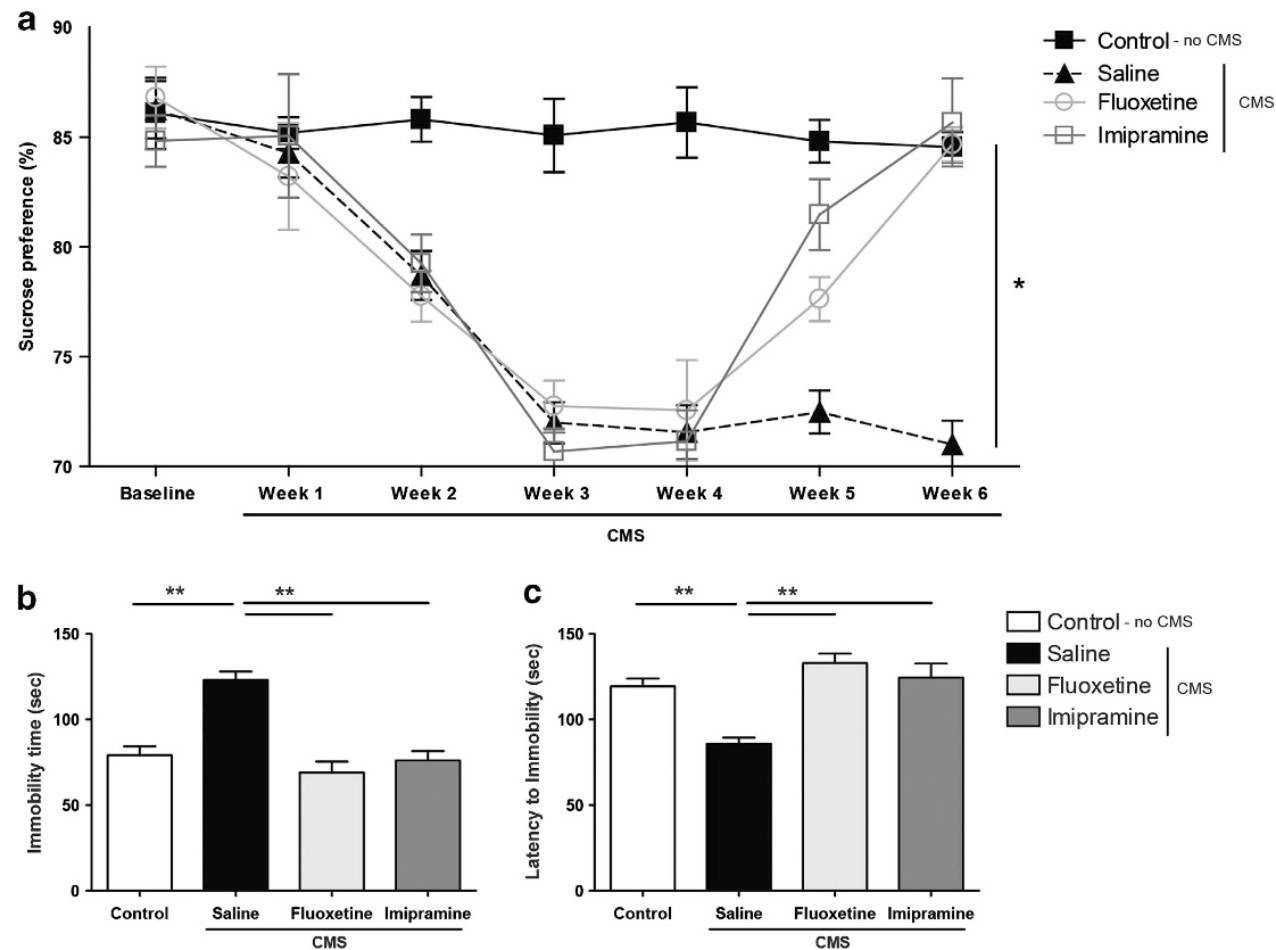

Figure 1 Behavioral effects of chronic mild stress (CMS) on hedonic and learned helplessness behavior. (a) Sucrose preference in the sucrose-consumption tests performed during CMS. (b) Immobility time and (c) latency to immobility time in the forced swimming test performed after CMS and drug administration. ${ }^{*} P<0.005$; ${ }^{* *} P<0.001$. Data presented as mean \pm s.e.m. 


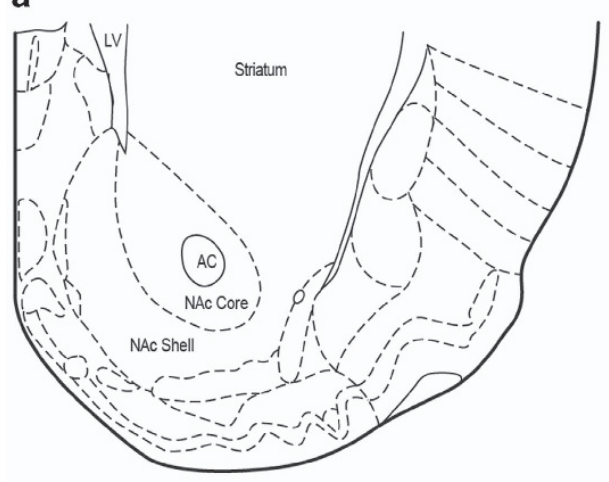

b

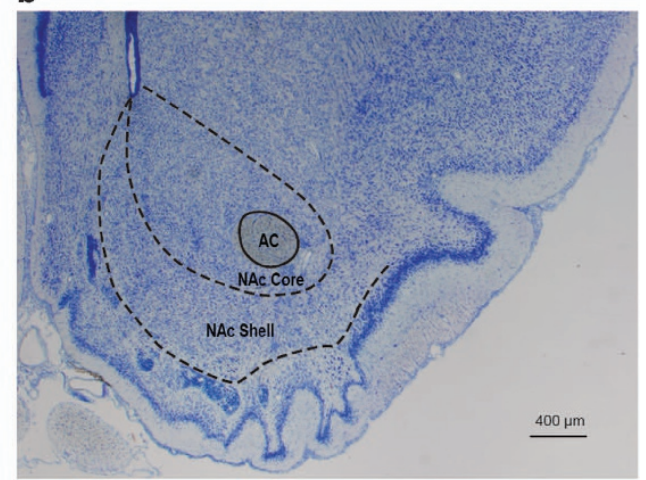

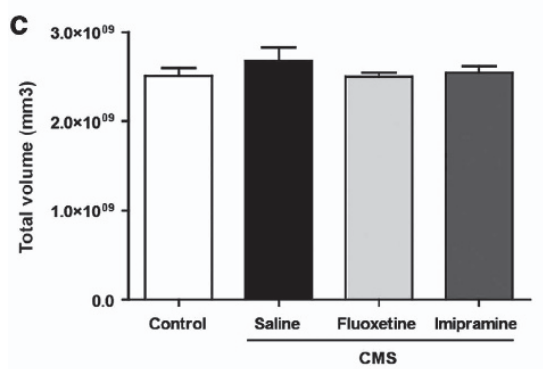
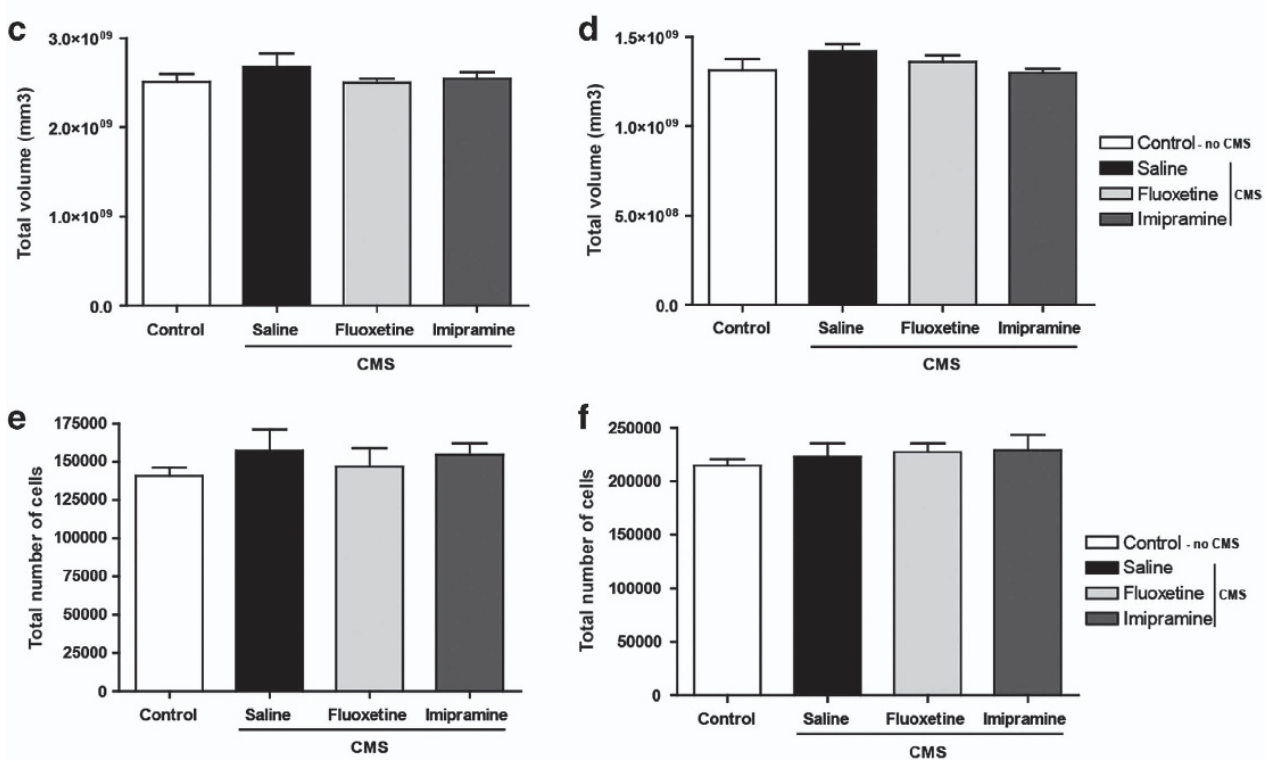

Figure 2 Stereological volumetric measurements and estimation of total number of neurons. (a) Paxinos atlas scheme, figure 56 (Bregma 1.8). (b) Low-power micrograph of a glycolmethacrylate-embedded brain section stained with Giemsa. Volumetric measurements of the core (c) and shell (d) divisions of the nucleus accumbens (NAc). Stereological estimation of the total number of cells in the core (e) and shell (f) divisions of the NAc. AC, anterior comissure; CMS, chronic mild stress; LV, lateral ventricle.

also did not reveal significant differences between experimental groups (shell: $P=0.770$; core: $P=0.666$ ) (Figures 2e and f).

On the other hand, 3D morphometric analysis of Golgiimpregnated neurons revealed that exposure to CMS induced dendritic hypertrophy in medium spiny neurons, with significant increase in their total dendritic length in the core $\left(F_{1,10}=7.195, P=0.023\right)$ and shell $\left(F_{1,10}=8.148, P=0.017\right)$ divisions of the NAc (Figures $3 \mathrm{~g}$ and $\mathrm{h}$ ). Importantly, this hypertrophic effect of stress was reversed after administration of the antidepressants fluoxetine (core: $P<0.001$; shell: $P=0.021$ ) and imipramine (core: $P=0.001$; shell: $P=0.041$ ).

Furthermore, exposure to CMS resulted in a significant increase in spine density in the core division of the NAc $\left(F_{1,10}=7.903, P=0.018\right)$ (Figure $\left.4 b\right)$. This gain in synaptic contacts was reversed by administration of fluoxetine $(P=0.016)$ and imipramine $(P=0.002)$. No significant changes were observed in spine densities in the shell division of the NAc (Figure 4c). None of the treatments led to significant differences in the relative abundance of mature (mushroom) and immature (thin) spines in the core and shell divisions of the NAC (Figures $4 d$ and e).
Gene-expression studies. The mRNA expression of Bdnf, Ncam1 and Syn1 was measured by qRT-PCR. The expression levels of $B d n f$ were significantly increased in the NAc $\left(F_{1,6}=8.794, P=0.025\right)$ of animals exposed to CMS. These changes were reversed after the administration of fluoxetine $(P=0.003)$ and imipramine $(P=0.021)$ (Figure 5a). Additionally, the CMS group revealed significantly increased Ncam1 expression in the NAc $\left(F_{1,6}=10.767, P=0.017\right)$ that was reversed by fluoxetine $(P=0.001)$ and imipramine $(P=0.027)$ (Figure $5 \mathrm{~b})$. Similarly, the expression levels of Syn1 were significantly increased in the NAc of rats exposed to CMS $\left(F_{1,6}=6.771, P=0.041\right)$ and reversed by administration of both fluoxetine $(P=0.039)$ and imipramine $(P=0.039)$ (Figure 5c).

\section{Discussion}

The brain regions involved in the genesis of the psychopathological phenomena associated with depression are not completely identified. Many studies have suggested a role for the NAc in depression as anhedonia is a cardinal symptom 
Control

a

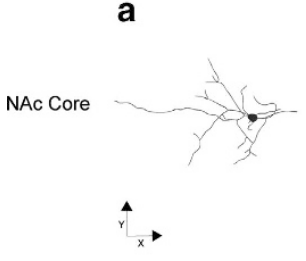

b

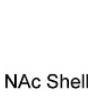

CMS

C

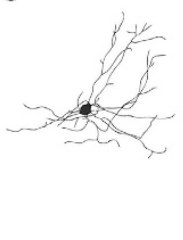

d

CMS + Antidepressants

e

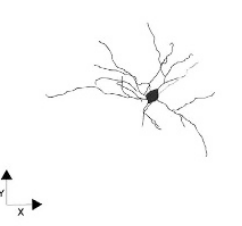

f

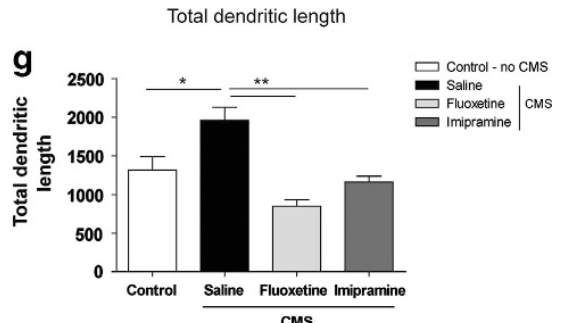

h

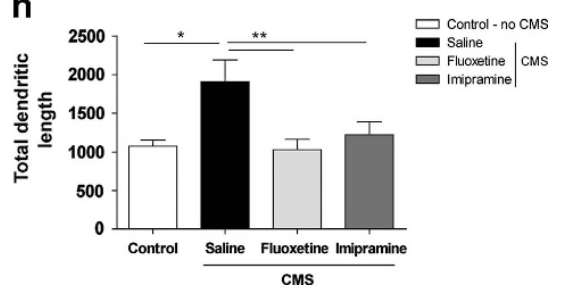

Figure 3 3D morphometric analysis of Golgi-impregnated neurons using computer- assisted reconstructions of nucleus accumbens (NAc) medium spiny neurons. Representative neurons of different experimental groups in the core (a, c, e), and shell (b, $\mathbf{d}, \mathbf{f})$ divisions of the NAc. Cells are depicted in the XY orthogonal plan. Total dendritic length of neurons in the core $(\mathbf{g})$ and shell $(\mathbf{h})$ divisions of the NAc. Asterisk represents the comparison between the control and chronic mild stress (CMS) groups. Double asterisk represents the effects of antidepressant treatment. ${ }^{\star} P<0.03$, ${ }^{*} P<0.03$. Data represented as mean \pm s.e.m.

a

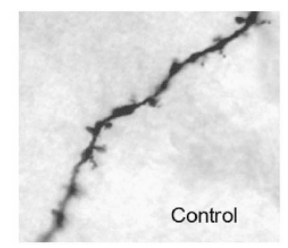

b

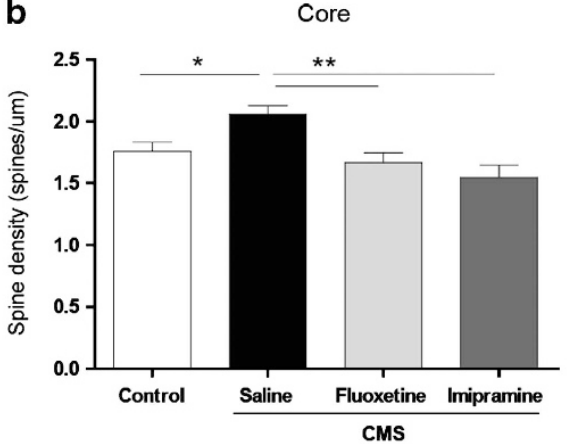

d

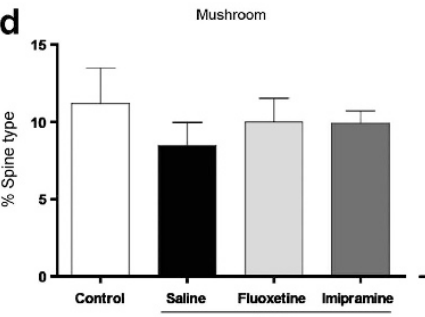

cms
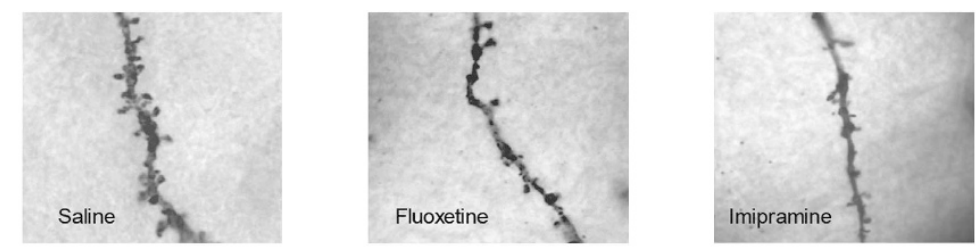

c

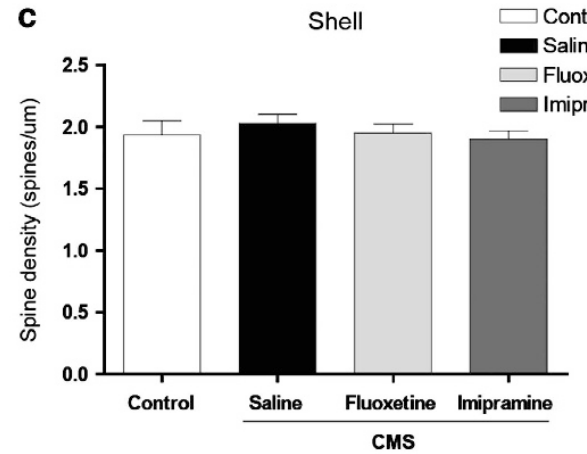

e

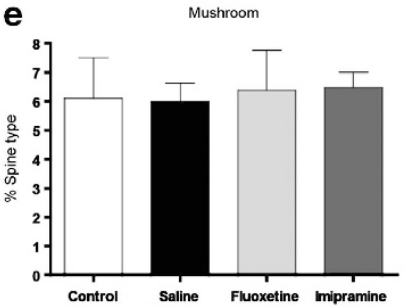

CMS

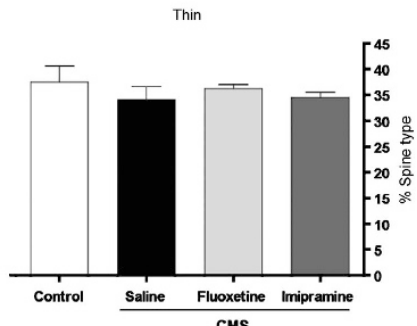

cus

Figure 4 Spine density and morphological classification. Representative dendritic segments for spine analysis from each experimental group (a). Spine densities of nucleus accumbens (NAc) medium spiny neurons in the core (b) and shell (c) divisions of the NAc. Morphological classification of dendritic spines in the core (d) and shell (e) subregions of NAc. Asterisk represents the comparison between the control and chronic mild stress groups. Double asterisk represents the effects of antidepressant treatment. ${ }^{\star} P<0.02,{ }^{*} P<0.02$. Data represented as mean \pm s.e.m.

in this condition, and the NAc is implicated in the regulation of motivation and reward processing. ${ }^{1-5}$ The results of the present study add important new support to this view.
Specifically, they show that exposure to unpredictable CMS, an established mean to induce depression-like behavior in rodents, increases anhedonic behavior (reduced sucrose 

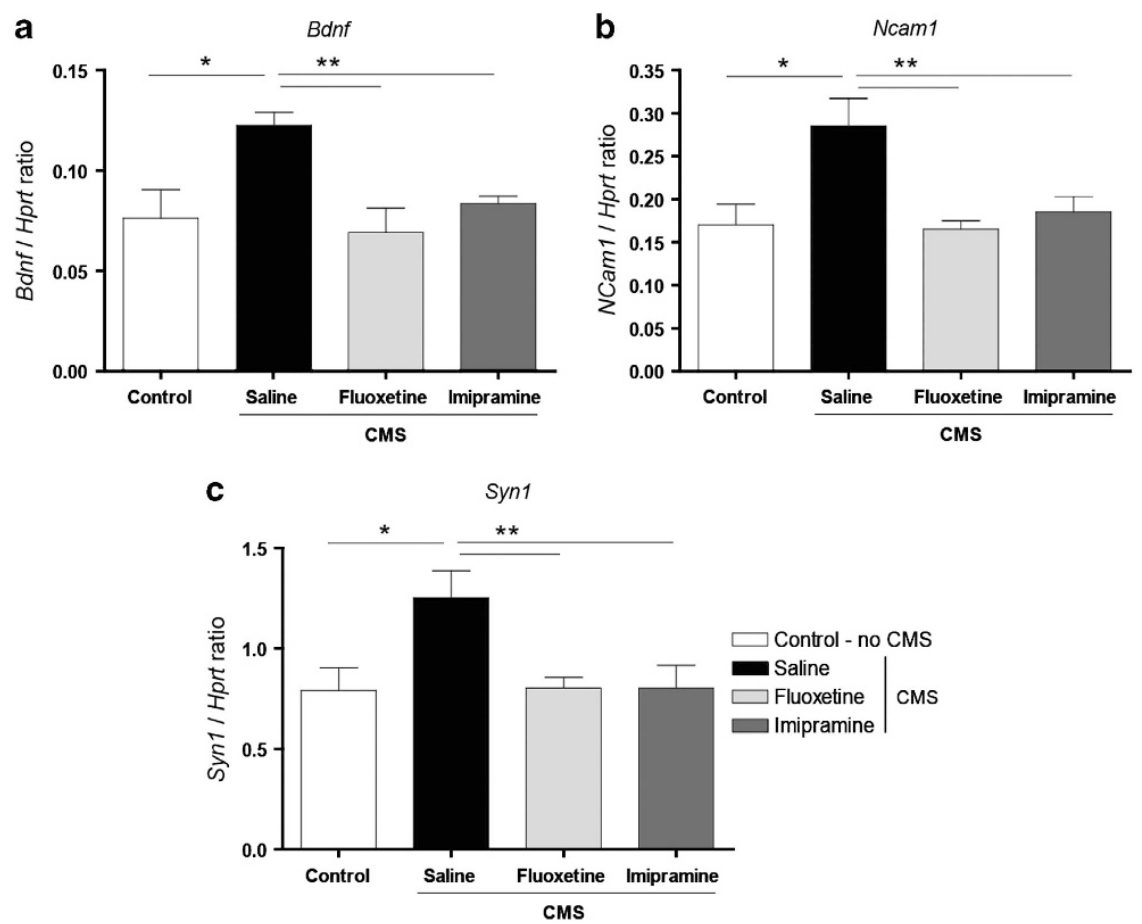

Figure 5 mRNA expression levels of (a) Bdnf (brain-derived growth factor), (b) Ncam1 (neural cell adhesion molecule 1) and (c) Syn1 (synapsin 1) in the nucleus accumbens measured by quantitative real-time PCR. All the genes analyzed showed an increased expression level in response to chronic mild stress (CMS), this effect being abrogated by the antidepressant treatment. Asterisk represents the comparison between control and CMS groups. Double asterisk represents the effects of antidepressant treatment. ${ }^{\star} P<0.05,{ }^{\star \star} P<0.05$. Data represented as mean \pm s.e.m. Hprt, hypoxanthine guanine phosphoribosyl transferase.

preference) in association with changes in the fine structure of the NAc. Although not affecting volumes or neuronal numbers in either the core or the shell of the NAc, chronic stress caused hypertrophy of the dendritic trees (increased dendritic lengths) and increased spine densities in the core division of the NAc. Importantly, the latter morphological adaptations were reversed by treatment with the tricyclic antidepressant imipramine as well as by the serotonin reuptake inhibitor fluoxetine; contemporaneously, these drugs attenuated the behavioral deficits seen in stress-exposed animals. Together, these observations add to the evidence that neuroplastic changes in the NAc contribute to the pathophysiology of depression and its pharmacologically induced recovery and point to the role of the NAc in the regulation of (an)hedonia.

The morphological findings in the NAc are interesting in that they contrast to the effects of chronic stress in the hippocampal formation and prefrontal cortex ${ }^{14,25}$ that have been the focus of most earlier studies aimed at identifying the neuroanatomical and neurochemical substrates of depression; in both areas, chronic stress leads to significant dendritic atrophy. ${ }^{20}$ On the other hand, stress has been shown previously to induce dendritic hypertrophy in other corticolimbic structures implicated in the regulation of mood and affect, namely, the amygdala, ${ }^{26}$ bed nucleus of stria terminals, ${ }^{27}$ dorsolateral striatum and orbitofrontal cortex, ${ }^{28}$ and to increase dendritic spine density and functional excitatory synaptic plasticity in the NAc. ${ }^{7,29}$ These observations reflect the complex neural networks that underlie the cluster of depressive symptoms. ${ }^{13}$

Stress-induced increases in dendritic arborization in the extended amygdala were previously ascribed to increases in
BDNF expression. ${ }^{30}$ Importantly, the stress-induced increases in spine density and synaptic plasticity in the NAC are dependent on the activity of the inhibitor of KB kinase, a downstream target of BDNF. ${ }^{31}$ Here, we also observed an upregulation in BDNF mRNA levels that was concomitant with dendritic hypertrophy in the NAc of stressed rats; moreover, both events were reverted by the antidepressants fluoxetine and imipramine. Here it is important to note that, although the recent work by Malenka and colleagues dissociates NAcmediated anhedonic behavior from behavioral despair, ${ }^{17}$ learned helplessness evaluated in the FST can be triggered by intra-VTA (ventral tegmental area) BDNF and blocked by overexpression of a dominant-negative mutant form of the BDNF receptor, TrkB. ${ }^{32}$ In addition, increased BDNF signalling in the VTA-NAc pathway has also been implicated in the display of other depression-like behaviors (decreased sexual behavior, decreased social interaction and a general decrease in locomotor activity), most of which are reversible by antidepressant treatment. Further, mice with selective knockdown of $B D N F$ in the mesolimbic pathway fail to develop depression-like behavior after exposure to social defeat stress. ${ }^{16}$ Thus, it would appear that an imbalance in BDNF signalling in the NAc contributes to dysregulated mood by altering hedonic tonus. Interestingly, recent evidences suggest a similar modulatory effect of BDNF in addictive behavior, where the suppression of BDNF in the mesolimbic pathways seems to increase dopaminergic neuron excitability and promote reward. ${ }^{33}$

An interesting outcome of the present work is the confirmation that some of the same molecular pathways implicated in depression and antidepressant action in the hippocampus and 
in the prefrontal cortex also influence these phenomena at the level of the NAc, although very different effects are seen in these various regions. The stress-induced changes in behavior are not only accompanied by increased BDNF expression but also by alterations in the expression profiles of other genes implicated in neuroplasticity. We report here that chronic stress upregulates the levels of both NCAM and SYN1, changes that are commensurate with the observed increases in dendritic length and synaptic contacts of medium spiny neurons of the NAc. Given that this region is remarkably interconnected with the hippocampus and the prefrontal cortex, it remains to be demonstrated whether the changes herein described are causal or a mere consequence of the neurodegenerative effects triggered by stress in these 'cortical' regions. Interestingly, however, the orbitofrontal cortex, an area also highly connected with the NAc, displays hypertrophic changes that are concordant with the present findings, which may suggest that these changes may be circuit specific. ${ }^{28}$

In conclusion, the present results provide a clear demonstration of structural changes in the NAc, a central component of the mesolimbic pathway, in stressed animals displaying anhedonic behavior. Of notice, these structural changes are reversible after efficient antidepressant treatment and may relate with the altered expression of molecules involved in neuroplasticity. These observations pave the way for future studies addressing whether primary abnormalities in the reward circuitry are involved in the etiology of depression and whether antidepressants operate through direct actions in the mesolimbic pathway or indirectly through other brain regions.

\section{Conflict of interest}

The authors declare no conflict of interest.

Acknowledgements. The present work was funded by the Portuguese Foundation for Technology (FCT), project PTDC/SAU-NEU/105180/2008. FM and $\mathrm{PL}$ are recipients of postdoctoral fellowships and MM is recipient of a doctoral fellowship, all from FCT, Portugal.

1. Gorwood P. Neurobiological mechanisms of anhedonia. Dialogues Clin Neurosci 2008; 10 291-299.

2. Wise RA. Neuroleptics and operant behavior: the anhedonia hypothesis. Behav Brain Sci 1982; 5: 39-87.

3. Di Chiara G, Loddo P, Tanda G. Reciprocal changes in prefrontal and limbic dopamine responsiveness to aversive and rewarding stimuli after chronic mild stress: implications for the psychobiology of depression. Biol Psychiatry 1999; 46: 1624-1633.

4. Newton SS, Thome J, Wallace TL, Shirayama Y, Schlesinger L, Sakai N et al. Inhibition of CAMP response element-binding protein or dynorphin in the nucleus accumbens produces an antidepressant-like effect. J Neurosci 2002; 22: 10883-10890.

5. Rada P, Moreno SA, Tucci S, Gonzalez LE, Harrison T, Chau DT et al. Glutamate release in the nucleus accumbens is involved in behavioral depression during the Porsolt swim test. Neuroscience 2003; 119: 557-565

6. Willner P. Chronic mild stress (CMS) revisited: consistency and behavioral-neurobiological concordance in the effects of CMS. Neuropsychobiology 2005; 52: 90-110.

7. Christoffel DJ, Golden SA, Russo SJ. Structural and synaptic plasticity in stress-related disorders. Rev Neurosci 2011; 22: 535-549.

8. Nestler EJ, Carlezon WA Jr. The mesolimbic dopamine reward circuit in depression. Biol Psychiatry 2006; 59: 1151-1159.

9. Ito R, Robbins TW, Everitt BJ. Differential control over cocaine-seeking behavior by nucleus accumbens core and shell. Nat Neurosci 2004; 7: 389-397.
10. Cardinal RN, Cheung THC. Nucleus accumbens core lesions retard instrumental learning and performance with delayed reinforcement in the rat. BMC Neurosci 2005; 6: 9 .

11. Bassareo V, De Luca MA, Di Chiara G. Differential expression of motivational stimulus properties by dopamine in nucleus accumbens shell versus core and prefrontal cortex. J Neurosci 2002; 22: 4709-4719.

12. Meredith GE, Pennartz CM, Groenewegen HJ. The cellular framework for chemical signalling in the nucleus accumbens. Prog Brain Res 1993; 99: 3-24.

13. Nestler EJ, Barrot M, DiLeone RJ, Eisch AJ, Gold SJ, Monteggia LM. Neurobiology of depression. Neuron 2002; 34: 13-25.

14. Bessa JM, Ferreira D, Melo I, Marques F, Cerqueira JJ, Palha JA et al. The moodimproving actions of antidepressants do not depend on neurogenesis but are associated with neuronal remodeling. Mol Psychiatry 2009; 14: 764-773.

15. Warner-Schmidt JL, Schmidt EF, Marshall JJ, Rubin AJ, Arango-Lievano M, Kaplitt MG et al. Cholinergic interneurons in the nucleus accumbens regulate depression-like behavior. Proc Natl Acad Sci 2012; 109: 11360-11365.

16. Berton O, McClung CA, DiLeone RJ, Krishnan V, Russo S, Graham D et al. Essential role of BDNF in the mesolimbic dopamine pathway in social defeat stress. Science 2006; 311: 864-868.

17. Lim BK, Huang KW, Grueter BA, Rothwell PE, Malenka RC. Anhedonia requires MC4Rmediated synaptic adaptations in nucleus accumbens. Nature 2012; 11: 183-189.

18. Song L, Che W, Min-Wei W, Murakami Y, Matsumoto K. Impairment of the spatial learning and memory induced by learned helplessness and chronic mild stress. Pharmacol Biochem Behav 2006; 83: 186-193.

19. Bekris S, Antoniou K, Daskas S, Papadopoulou-Daifoti Z. Behavioral and neurochemical effects induced by chronic mild stress applied to two different rat strains. Behav Brain Res 2005; 161: 45-59.

20. Cerqueira JJ, Mailliet F, Almeida OF, Jay TM, Sousa N. The prefrontal cortex as a key target of the maladaptive response to stress. J Neurosci 2007; 27: 2781-2787.

21. Gibb R, Kolb B. A method for vibratome sectioning of Golgi-Cox stained whole rat brain. J Neurosci Methods 1998; 79: 1-4.

22. Glaser EM, Van der Loos H. Analysis of thick brain sections by obverse-reverse computer microscopy: application of a new, high clarity Golgi-Nissl stain. $J$ Neurosci Methods 1981; 4: 117-125.

23. Harris KM, Jensen FE, Tsao B. Three-dimensional structure of dendritic spines and synapses in rat hippocampus (CA1) at postnatal day 15 and adult ages: implications for the maturation of synaptic physiology and long-term potentiation. J Neurosci 1992; 12: 2685-26705.

24. Bessa JM, Mesquita A, Oliveira M, Pêgo JM, Cerqueira JJ, Palha JA et al. A transdimensional approach to the behavioural aspects of depression. Front Behav Neurosci 2009; 3 : 1.

25. Dranovsky A, Hen R. Hippocampal neurogenesis: regulation by stress and antidepressants. Biol Psychiatry 2006; 59: 1136-1143.

26. Vyas A, Bernal S, Chattarji S. Effects of chronic stress on dendritic arborization in the central and extended amygdala. Brain Res 2003; 965: 290-294.

27. Pêgo JM, Morgado P, Pinto LG, Cerqueira JJ, Almeida OF, Sousa N. Dissociation of the morphological correlates of stress-induced anxiety and fear. Eur J Neurosci 2008; 27: 1503-1516.

28. Dias-Ferreira E, Sousa JC, Melo I, Morgado P, Mesquita AR, Cerqueira JJ et al. Chronic stress causes frontostriatal reorganization and affects decision-making. Science 2009; 325: 621-625.

29. Christoffel DJ, Golden SA, Dumitriu D, Robison AJ, Janssen WG, Ahn HF et al. I $\mathrm{kB}$ kinase regulates social defeat stress-induced synaptic and behavioral plasticity. J Neurosci 2011; 31: 314-321.

30. Reagan LP, Hendry RM, Reznikov LR, Piroli GG, Wood GE, McEwen BS et al. Tianeptine increases brain-derived neurotrophic factor expression in the rat amygdala. Eur $J$ Pharmacol 2007; 565: 68-75.

31. Christoffel DJ, Golden SA, Heshmati M, Graham A, Birnbaum S, Neve RL et al. Effects of inhibitor of $\mathrm{\kappa B}$ kinase activity in the nucleus accumbens on emotional behavior. Neuropsychopharmacology 2012; 37: 2615-2623.

32. Eisch AJ, Bolanos CA, Wit JD, Simonak RD, Pudiak CM, Barrot M et al. BDNF in the ventral midbrain-nucleus accumbens pathway: a role in depression. Biol Psychiatry 2003; 54: 994-1005.

33. Koo JW, Mazei-Robison MS, Chaudhury D, Juarez B, LaPlant Q, Ferguson D et al. BDNF is a negative modulator of morphine action. Science 2012; 338: 124-128.

Translational Psychiatry is an open-access journal (C) ${ }_{\mathrm{B}}$ sa
licensed under a Creative Commons Attribution-NonCommercialShareAlike 3.0 Unported License. To view a copy of this license, visit http://creativecommons.org/licenses/by-nc-sa/3.0/ 